\title{
TURN-BY-TURN BPM ELECTRONICS BASED ON 500 MHz LOG-RATIO AMPLIFIER
}

\author{
K.H. Hu, Jenny Chen, C. H. Kuo, K.T. Hsu, T. S. Ueng \\ Synchrotron Radiation Research Center \\ No 1, R\&D Road VI, Hsinchu Science-Based Industrial Park, Hsinchu, Taiwan, R.O.C.
}

\begin{abstract}
Log-ratio processor working at $500 \mathrm{MHz}$ applied to turn-by-turn beam position measurement was implemented. The processor circuit consists of two logarithmic amplifiers and a subtraction circuit. The output value is proportional to beam position. Before, the upper usable frequency of logarithmic amplify is lower than 500MHz. At present, several manufacturers support higher upper usable frequency of logarithmic amplifiers. In this paper, we will present the performance test result of the $500 \mathrm{MHz}$ log-ratio processor applied to turn-by-turn BPM electronics.
\end{abstract}

\section{INTRODUCTION}

Wells and Gilpatrik et al. [1,2] have mentioned logratio processor working at $100 \mathrm{MHz}$ to $500 \mathrm{MHz}$. Most of the log-ratio processors need down converter to translate interested frequency to this working intermediate frequency $(100 \mathrm{KHz} 100 \mathrm{MHz})$. Electronics working at bunch-crossing frequency can reduce the complexity of signal processing chain. Benefited from the promising progress in wireless communication recently, low cost wide bandwidth logarithmic amplifier is available. The BPM electronics based upon this complete multistage logamplifier is highly interesting due to its low cost and simple. The bunch-crossing rate of SRRC storage ring is $500 \mathrm{MHz}$. The turn-by-turn beam position signal processed by using log-ratio processor directly working at $500 \mathrm{MHz}$ is reported here. This system is working at single bunch or multi-bunch operation mode. Two versions of amplifiers have been tested. One is a commercial log-amplifier module, another is using complete multistage log-amplifier chip. The preliminary test result is presented here.

\section{LOG-RATIO ELECTRONICS FOR TURN-BY- TURN BEAM POSITION DETECTION}

The logarithmic amplifier feature includes large input dynamic range, bandwidth $500 \mathrm{MHz}$ or more, easy to use, low cost, and so on. The detection signal at RF frequency provide a simple solution compared with difference-oversum or AM/PM conversion approaches. Normally, logarithmic amplifier applied to a very wide range IF and RF power measurement. The logarithmic amplifier and a subtraction circuit (sum, difference, ...etc) have been applied to beam position / intensity measurement module [2][3]. A logarithmic amplifier function is expressed as equation (1).

$\mathrm{V}_{\text {OUT }}=\mathrm{V}_{\text {SLOP }} \log \left(\mathrm{P}_{\mathrm{IN}} / \mathrm{P}_{\mathrm{O}}\right)$. where:

$\mathrm{V}_{\text {OUT }}$ is the demodulated and filtered output.

$\mathrm{V}_{\mathrm{SLOPE}}$ is the logarithmic slop (V/dB).

$\mathrm{P}_{\mathrm{IN}}$ is input power.

$\mathrm{P}_{\mathrm{O}}$ is the logarithmic intercept.

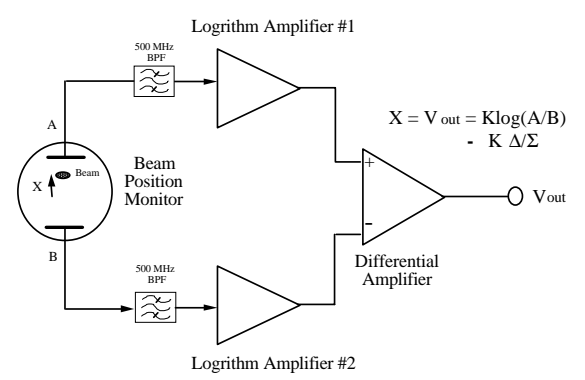

Figure 1: 500MHz log-ratio processor.

The $500 \mathrm{MHz}$ log-ratio processor consists of the 500 $\mathrm{MHz}$ band-pass filter and two logarithmic amplifiers and a differential amplifier as show in Fig. 1. Log-ratio processor output direct proposition to normalize beam position $\mathrm{X}$ is expressed in equation (2).

$$
\begin{aligned}
\mathrm{X} & =\mathrm{K}(\Delta / \Sigma) \fallingdotseq \mathrm{K} \log (\mathrm{A} / \mathrm{B}) \\
& =\mathrm{V}_{\text {OUT }}
\end{aligned}
$$

The revolution of storage ring is $2.5 \mathrm{MHz}$. BPMs, hybrid junctions, 500MHZ band-pass filters $(15 \mathrm{MHz}$ bandwidth) and log-ratio processor make up the turn-byturn measurement system.

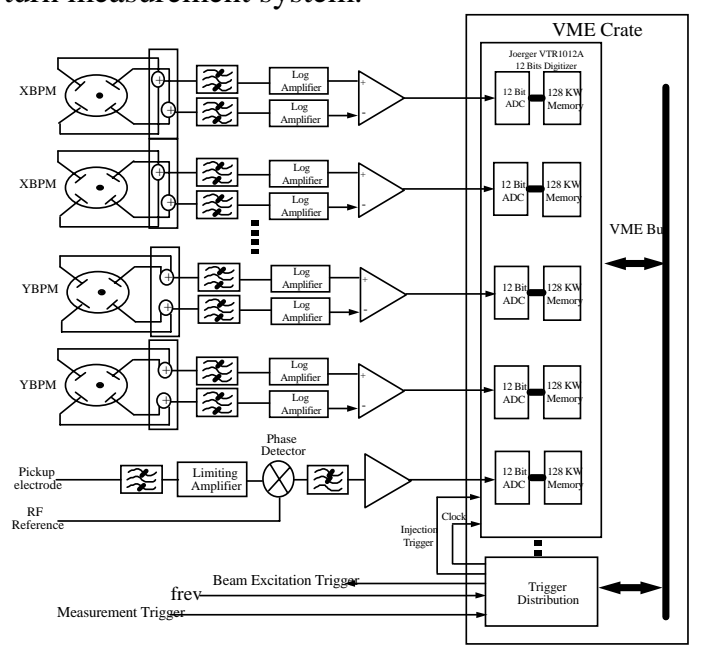

Figure 2: Block diagram of the turn-by-turn BPM system. 
The button electrode of BPM is mounted on skew position. Hybrid junctions were used to transform the BPM as horizontal or vertical direction BPM. The stored beam induces an impulse like doublet at button electrode. After the hybrid junctions, the output of BPF is impulse response with $500 \mathrm{MHz}$ center frequency. The electron beam signal induced by the BPM buttons to pass through hybrid junction and the log-ratio processor to VME based transient digitizer. The clock of the transient digitizer is the revolution clock. The digitizer records the turn-by-turn beam position. The system is useful for studying the nonlinear beam dynamics. In this report we attempt to use two versions of logarithmic amplifiers. One is a commercial log-amplifier module, which was made by discreet components. Another is to use the log-amplifier chip. Results of both versions of log-ratio processors are similar.

\section{PRELIMINARY BEAM TEST RESULTS}

Implement the turn-by-turn BPM electronics by using $500 \mathrm{MHz} \log$-ratio processor is the goal of this study. The preliminary beam test was done to evaluate the performance of log-ratio processor. The turn-by-turn beam position measurement of a stable beam was used to estimate resolution of processing electronics. Fig. 3 shows the beam position data is scattered in a small region. The resolution of electronics is estimated about $100 \mu \mathrm{m}$ when beam current is $10 \mathrm{~mA}$. The processor can keep its resolution with the stored beam down to $1 \mathrm{~mA}$. For a routine use, the further improvement is needed.

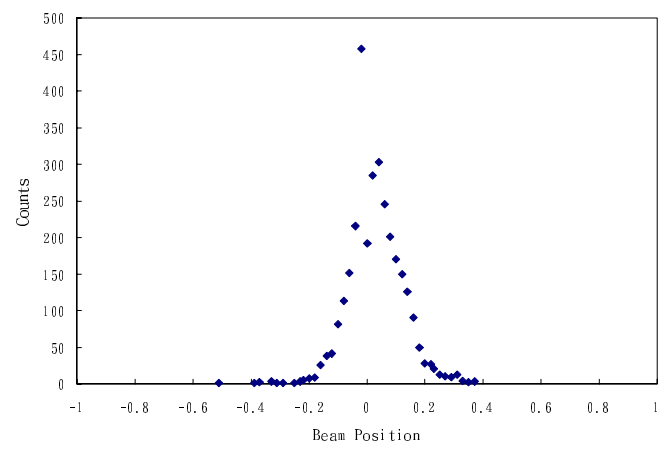

Figure3: Histogram of the beam position reading when beam is stable.

One of an injection kicker was used to excite horizontal betatron oscillation of the stored beam, the kick angle is about $1 \mathrm{mrad}$, and the beam will undergo damped oscillation as shown in Fig. 4. The horizontal tune at measurement is 7.196. The damping is strong which is lattice dependent. From the measured data, it confirms that the system is working well in turn-by-turn BPM signal processing at the bunch crossing frequency.

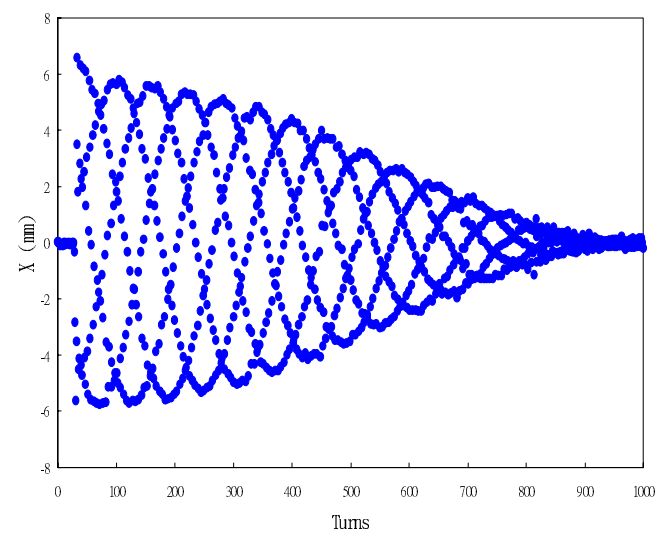

Figure 4: Horizontal turn-by-turn beam position, $v \mathrm{x}=7.196$

To explore more depth, we set the tune near fourth order resonance $\left(4 v_{x}=29\right)$. The turn-by-turn beam position and the phase space plot are shown in Fig. 5 and Fig. 6. The kick strength is about $1.5 \mathrm{mrad}$ in this measurement. The spiral like arm damp rapidly. De-coherence and recoherence is observed from the turn-by-turn beam position data.

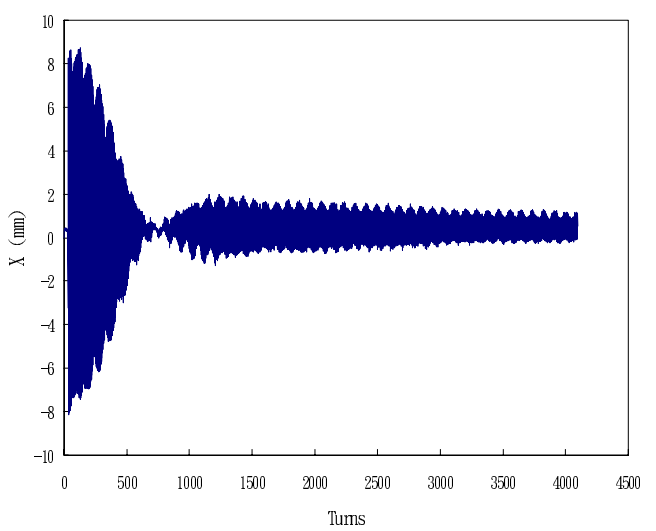

Figure 5: Horizontal turn-by-turn beam position, near $4 v_{x}$ $=29$ resonance.

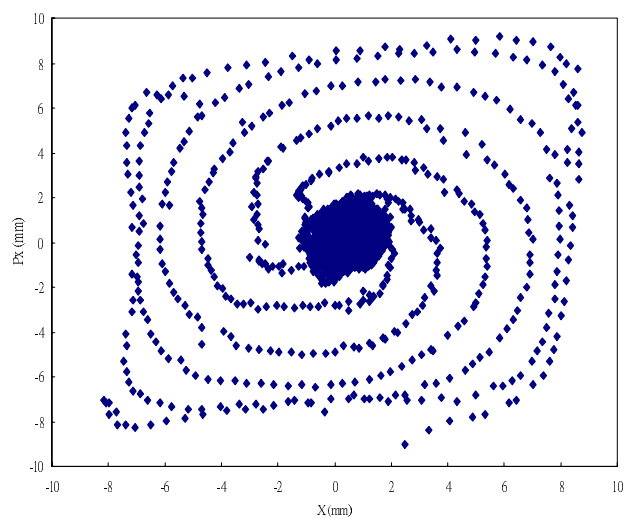

Figure 6: Phase space plot, near $4 v_{x}=29$ resonance. 


\section{PERFORMANCE TEST OF LOG-AMP CHIPS}

Various measurements are performed to familiarize the behavior of log-amplifier chips working at $500 \mathrm{MHz}$ [5]. The output voltage as a function of input power is shown in Fig. 7. The slope is $20 \mathrm{mV} / \mathrm{dB}$. The log linearity of the chip working at $500 \mathrm{MHz}$ is shown in Fig. 8. The output linearity is less than $\pm 0.5 \mathrm{~dB}$ without correction. The linearity is acceptable for BPM signal processing. Over 70 $\mathrm{dB}$ dynamic range $(-70 \mathrm{dBm} \sim 10 \mathrm{dBm})$ is also applicable in BPM application.

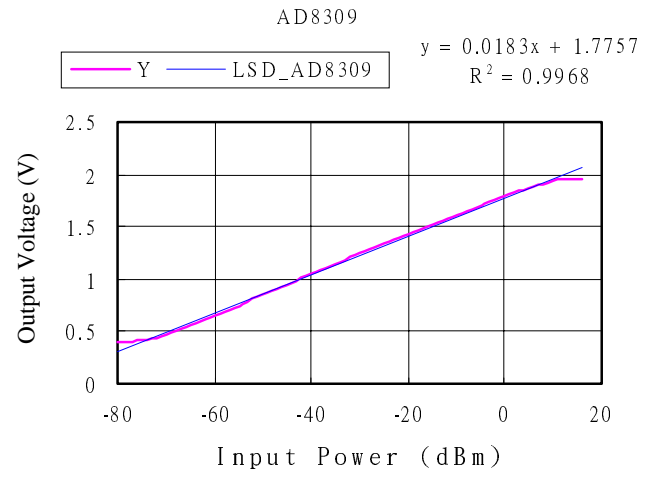

Figure 7: Output vs. input power, Frequency for $500 \mathrm{MHz}$, Single-Ended Input.

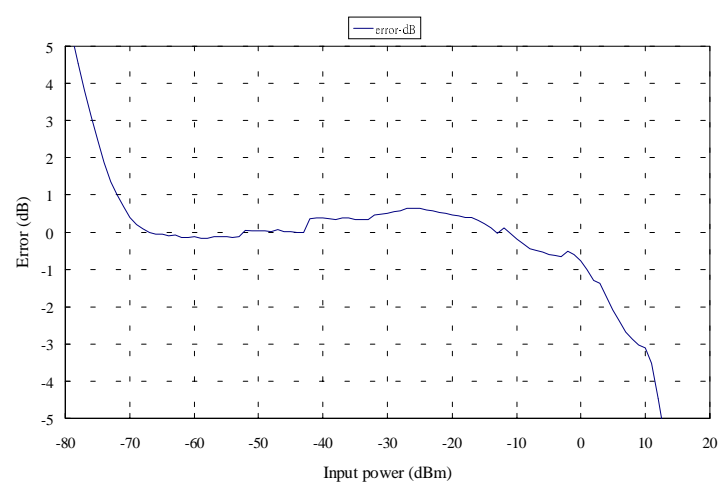

Figure 8: Log linearity of logarithmic amplifier output vs. input power, at frequency of $500 \mathrm{MHz}$.

$\mathrm{o}(0,-5) \ldots . . . \mathrm{o}(-5,0)$

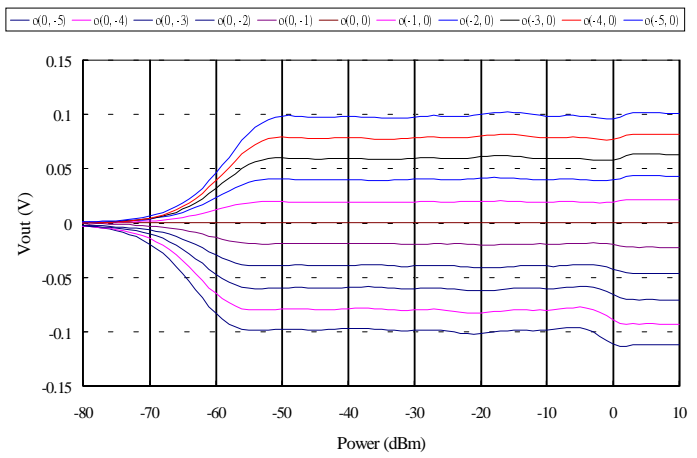

Figure 9: The output voltage offset of the Log-ratio processor when difference input power.
The preliminary test of log-amplifier chip (AD8309) based log-ratio processor was used for this test. The corrected data is shown in Fig. 9. The vertical scale is in voltage, and the horizontal scale is in $\mathrm{dBm}$. The input $\mathrm{RF}$ signals to both channels are offset by $-5 \mathrm{dBm}$ to $5 \mathrm{dBm}$. Important characteristics to note in the graph of Fig. 8 is that the curve exhibit the dynamics range of the test. For a properly performing log-ratio system, the line will be centered about zero offset and be flat. The performance will be improved after a special care during the layout.

\section{SUMMARY}

This paper presents a log-ratio processor working at $500 \mathrm{MHz}$ for a turn-by-turn beam position measurement that eliminates the need of down-converter. Preliminary results showed $100 \mu \mathrm{m}$ resolution was achievable with simple electronics. Commercial logarithmic amplifier chips (e.g. AD8309) which could work at $500 \mathrm{MHz}$ directly are highly interested. The test of usability of AD8309 for $500 \mathrm{MHz}$ application was also performed. The new electronics for the beam position monitor on the boosterto-storage ring transport line will be installed with dedicated log-ratio processors soon.

\section{REFERENCES}

[1] F. D. Wells, et al., "Beam Position Monitoring in the $100 \mathrm{MHz}$ to $500 \mathrm{MHz}$ Frequency Using the Log-Ratio Technique”, PAC'93, Washington, 2316 (1993).

[2] J. D. Gilpatrick, et al., "LEDA \& APT Beam Position Measurement System: Design and Initial Tests", LINAC'98, Chicago, Il., August 23-28, 1998.

[3] C. R. Rose, et al., "Description and Operation of the LEDA Beam Position/Intensity Measurement Module”, PAC'97, Vancouver, BC, Canada, June 1997.

[4] C. R. Rose, et al., "Test Results of the LEDA Beam Position /Intensity Measurement Module", BIW'98, Stanford, CA, May 4-7, 1998, pp 423-427.

[5] Data sheet of AD8309, Analog Devices, Inc., 1998. 